Results The most important clinical factor influencing patient outcome was the colorectal cancer itself, and hence there was no significant difference between five year OS (55\%), TTP (62\%) and PFS (52\%). As expected the disease was most likely to recur in subjects with more advanced tumours (Duke's C $p=0.04$ ) and male sex. However, irrespective of the tumour stages Duke's A-C, the most significant risk factor for tumour recurrence was the presence of anti-CEA CD4 ${ }^{+} \mathrm{T}$ cell responses, the majority of which were suppressed by Tregs $(p=0.002)$. The magnitude of these responses was greater in the group with disease recurrence $(p=0.004)$. Pre-operative responses to other antigens, including the tumour antigen 5T4, did not reflect outcome.

Conclusion The presence of pre-operative anti-CEA immune responses identifies patients most likely to experience CRC recurrence during the 5 year follow-up period. This relationship holds true irrespective of the tumour stage. This information might be used to direct adjuvant treatment strategies.

Disclosure of Interest None Declared.

\section{PWE-152 SELECTIVE LOSS OF ONCOFOETAL ANTIGEN 5T4-SPECIFIC T CELL RESPONSE CORRELATES WITH PROGRESSION OF COLORECTAL CANCER}

doi:10.1136/gutjnl-2013-304907.440

${ }^{1} \mathrm{M}$ Scurr, ${ }^{2} \mathrm{M}$ Davies, ${ }^{2} \mathrm{~S}$ Phillips, ${ }^{2 \mathrm{R}}$ Hargest, ${ }^{3} \mathrm{~A}$ Christian, ${ }^{3} \mathrm{~T}$ Hockey, ${ }^{3} \mathrm{G}$ Williams, 'A Gallimore, 1,"A Godkin. 'Institute of Infection and Immunity, Cardiff University; ${ }^{2}$ Department of Surgery; ${ }^{3}$ Department of Histopathology, Cardiff and Vale University Health Board, Cardiff, UK

Introduction The human oncofoetal antigen 5T4 is expressed on many human carcinomas, including colorectal cancer (CRC) cells, but has limited expression on normal tissues making it an ideal target for cancer immunotherapy. Here, a significant loss of $\mathrm{T}$ cell response to 5T4 in patients with more advanced CRC has been identified.

Methods Lymphocyte samples obtained from HLA-typed CRC patients and healthy donor controls were cultured for two weeks with pools of overlapping 20mer 5T4 peptides, spanning the entire protein, before subsequent analysis for antigen specificity, as measured by the highly sensitive IFN-g/IL-10 ELISPOT assay.

Results Positive 5T4-specific lines were identified in 79\% (15/19) of CRC patients and all (11/11) healthy donors tested. Intriguingly, CRC patients respond to significantly fewer candidate epitopes and generate a lower magnitude of IFN- $\gamma$ responses to 5T4. Furthermore this response diminishes with tumour advancement despite similar responses to the recall antigen PPD. The mechanism of loss of $T$ cell response is independent of HLA-DR type or patient age, but depletion experiments indicate suppression by Foxp3 ${ }^{+}$regulatory $\mathrm{CD}^{+}$ $T$ cells. In addition, analysis of peripheral blood and tumour-infiltrating lymphocytes in the same cohort of patients revealed a marked suppressive phenotype in comparison to healthy agematched controls.

Conclusion Effective anti-tumour immunotherapy will be reliant upon overcoming such regulation of tumour-specific $\mathrm{T}$ cell responses. These data support a rationale for re-stimulating 5T4-specific immune responses in CRC patients, and reducing tumour-induced immunosuppression to enhance immunotherapy.

Disclosure of Interest None Declared.

\section{PWE-153 ASSOCIATION BETWEEN CONSTIPATION AND COLORECTAL CANCER: SYSTEMATIC REVIEW AND META- ANALYSIS OF OBSERVATIONAL STUDIES}

doi:10.1136/gutjnl-2013-304907.441

1."A M Power, ${ }^{2} \mathrm{~N} J$ Talley, ${ }^{1}$ A C Ford. 'Leeds Gastroenterology Institute, St. James's University Hospital, leeds, Leeds, UK; ${ }^{2}$ Faculty of Health, University of Newcastle, Callaghan, Australia
Introduction Constipation is common in the community, and may affect survival adversely. An association between constipation and development of colorectal cancer (CRC) could be one possible explanation for this association. We performed a systematic review and meta-analysis examining this issue.

Methods We searched MEDLINE, EMBASE, and EMBASE Classic (through July 2012). Eligible studies were cross-sectional surveys, cohort studies, or case-control studies reporting the association between constipation and CRC. For cross-sectional surveys and cohort studies, we recorded number of subjects with CRC according to constipation status, and for case-control studies number of subjects with constipation according to CRC status. Study quality was assessed according to published criteria. Data were pooled using a random effects model, and the association between CRC and constipation was summarised using an odds ratio (OR) with a $95 \%$ confidence interval (CI).

Results The search strategy identified 2282 citations, of which 28 were eligible. In eight cross-sectional surveys, presence of constipation as the primary indication for colonoscopywas associated with a lower prevalence of CRC (OR 0.56; 95\% CI 0.36-0.89). There was a trend towards a reduction in odds of CRC in constipation in three cohort studies (OR $=0.80 ; 95 \%$ CI 0.61-1.04). The prevalence of constipation in CRC was significantly higher than in controls without CRC in 17 case-control studies (OR $=1.68 ; 95 \%$ CI 1.29-2.18), but with significant heterogeneity, and possible publication bias

Conclusion Conclusions: Prospective cross-sectional surveys and cohort studies demonstrate no increase in prevalence of CRC in patients or individuals with constipation. The significant association observed in case-control studies may relate to recall bias.

Disclosure of Interest None Declared.

\section{PWE-154 IRON DEFICIENCY ANAEMIA AS AN INDICATOR OF MALIGNANCY - THE IDIOM STUDY}

doi:10.1136/gutjnl-2013-304907.442

1,*A Castro-Silva, ${ }^{2}$ Z A Sheppard, 'S L Surgenor, 'E J Williams, 'J A Snook. 'Gastroenterology Unit, Poole Hospital NHS Foundation Trust, Poole; ${ }^{2}$ Clinical Research Unit, Bournemouth University, Bournemouth, UK

Introduction Iron deficiency anaemia (IDA) is common. It is of particular importance because about $10 \%$ of subjects with IDA over the age of 50 will have an underlying gastro-intestinal (GI) malignancy, often in the absence of other clinical pointers to the diagnosis. IDA is therefore an accepted indication for examination of the GI tract, generally through bidirectional endoscopy.

Investigation of IDA is labour-intensive however, and most examinations will not reveal significant pathology. The aim of this study was to determine whether simple and objective clinical variables can identify sub-groups of subjects with IDA who are at clinically useful extremes of risk for underlying malignancy - arbitrarily defined as $<1 \%$ for low risk and $>20 \%$ for high risk.

Methods A retrospective study of 720 subjects referred to a single IDA clinic between 2004 and 2012. All had confirmed iron deficiency, minor or no localising symptoms, and subsequent GI tract investigation. Recorded information included age, sex, haemoglobin concentration $(\mathrm{Hb})$, mean cell volume (MCV), iron studies, and final diagnosis.

Results A total of 68 (9.4\%) of the study population had a GI malignancy. In the model generated by logistic regression analysis, age $(>70 \vee<71)$, sex $(M \vee F)$ and $\mathrm{Hb}$ quartile were all predictive of the probability of underlying malignancy. The effects of these variables were cumulative.

Percentage of cases of GI malignancy in each subgroup (cases/ number in subgroup): 


\section{Abstract PWE-154 Table 1}

\begin{tabular}{llllll}
\hline Hb quartile & Hb g/l & $\mathbf{F}<\mathbf{7 1 y r s}$ & $\mathbf{F}>\mathbf{7 0 y r s}$ & $\mathbf{M}<\mathbf{7 1 y r s}$ & $\mathbf{M}>\mathbf{7 0 y r s}$ \\
\hline 1 & $111-158$ & $0.0 \%(0 / 35)$ & $2.6 \%(1 / 38)$ & $2.4 \%(1 / 42)$ & $10.8 \%(7 / 65)$ \\
2 & $102-111$ & $0.0 \%(0 / 49)$ & $3.7 \%(3 / 81)$ & $5.3 \%(1 / 19)$ & $19.4 \%(6 / 31)$ \\
3 & $91-102$ & $3.6 \%(2 / 55)$ & $8.8 \%(6 / 68)$ & $0.0 \%(0 / 13)$ & $25.0 \%(11 / 44)$ \\
4 & $42-91$ & $2.4 \%(1 / 41)$ & $13.6 \%(9 / 66)$ & $23.3 \%(7 / 30)$ & $30.2 \%(13 / 43)$ \\
\hline
\end{tabular}

The prevalence of GI malignancy ranged from $0.0 \%$ in younger females with mild anaemia, to over $25 \%$ in older males with more severe anaemia. By the pre-defined criteria, the model identified subpopulations of 84 (11\% of the total) at extreme low risk, and 117 $(16 \%)$ at extreme high risk.

Conclusion The results confirm previous work identifying age, sex and haemoglobin concentration as variables predictive of underlying malignancy in IDA. Furthermore, the findings suggest that over a quarter of subjects with IDA can be predicted to be of extremely low or high risk on the basis of these simple and objective clinical criteria. This may be of clinical relevance for patient counselling, prioritisation of investigations and allocation of resources. Work is ongoing to validate risk prediction in a prospective study, and to refine the model by inclusion of additional variables.

Disclosure of Interest None Declared.

\section{REFERENCE}

James MW, Chen CM, Goddard WP, Scott BB, Goddard AF. Risk factors for gastrointestinal malignancy in patients with iron deficiency anaemia. Eur J Gastroenterol Hepatol 2005; 17(11):1197-203.

\section{PWE-155 A BAD GUT FEELING: THE LONG-TERM IMPACT OF PELVIC RADIOTHERAPY ON GASTROINTESTINAL (GI) FUNCTION}

doi:10.1136/gutjnl-2013-304907.443

\section{1."A C Muls, 'A Lalij, 'H J Andreyev. ${ }^{1}$ ROYAL MARSDEN HOSPITAL, LONDON, UK}

Introduction As new cancer treatments have been introduced, there have been enormous improvements in outcomes for treated patients. They are living longer and the number of survivors of cancer therapy is growing by 3\% per year in the UK. 17000 UK patients are treated annually with pelvic radiotherapy. $80 \%$ of patients who receive pelvic radiotherapy are left with chronic alteration in GI function and $50 \%$ state that this affects daily activity. There are few data on the nature of the symptoms these patients develop. This study aims to describe the symptoms troubling patients referred to a specialist Pelvic Radiation Disease clinic.

Methods A prospective service evaluation of patients treated with pelvic radiotherapy referred to our clinic was performed. Patient characteristics were recorded. Each new patient completed a modified Gastrointestinal Symptom Rating Scale and Bristol Stool Chart which described their symptoms and severity.

Results Data on the first 110 patients collected included 47 women (43\%), median age, 59 (range: 37-79 years) and 63 men (57\%) median age, 72 years (range: 20-83 years) treated for prostate (47\%), gynaecological $(27 \%)$ or anorectal cancers $(17 \%)$, lymphoma $(5 \%)$ and other tumours (4\%). The median length of time since starting radiotherapy to presenting in clinic was 3 years 1 month; range: $0.5-36$ years.

Pelvic symptoms causing frequent or severe impact on patients daily lives were urgency (68\%), diarrhoea (defined as Bristol stool chart type 6 or 7 ) (62\%), tenesmus (55\%), fatigue (51\%), rectal flatulence $(51 \%)$, abdominal pain (45\%), faecal leakage (43\%), sexual concerns $(35 \%)$, problems with urination $(34 \%)$, bloating $(34 \%)$, borborygmi $(30 \%)$, woken at night to defaecate $(28 \%)$, rectal bleeding $(24 \%)$, belching $(20 \%)$, heartburn $(15 \%)$, steatorrhoea $(13 \%)$, nausea and vomiting (10\%).

Women presented with a median of 12 symptoms (range: 2-17) out of a maximum of 17 recorded symptoms and men with a median of 11 (range: 2-16). The number of symptoms defined by the patients as "frequent" or "severe" was a median of 8 symptoms for women (range: 0-15) and 5 symptoms for men (range: 0-13).

Conclusion GI, sexual and urinary symptom burden is high after pelvic irradiation in new patients attending our clinic. Patients often present with multiple symptoms impacting daily activities. Symptoms clusters are complex and a systematic, multidisciplinary approach for efficient management is required. Clinicians will see increasing numbers of affected patients and may need training to deal with these patients optimally.

Disclosure of Interest None Declared.

\section{PWE-156 GASTRO-0ESOPHAGEAL REFLUX DISEASE (GORD) SYMPTOMATOLOGY IS NOT A RELIABLE PREDICTOR OF OESOPHAGEAL ADENOCARCINOMA}

doi:10.1136/gutjnl-2013-304907.444

1.*A Sugumaran, 'A Rasheed. 'Gwent Centre for Digestive Diseases (GCDD), Royal Gwent hospital, Newport, UK

Introduction Chronic gastro-oesophageal reflux disease (GORD) is considered a risk factor for development of gastro-oesophageal junction adenocarcinoma. Our aim is to determine the prevalence of GORD symptomatology and Barrett's columnar metaplasia prior to the diagnosis of distal oesophageal, gastro-oesophageal junction (GOJ) and gastric cardia adenocarcinoma at GCDD over a 10 year period. Methods A prospective pilot study collected data from patients diagnosed with adenocarcinomas arising from the distal oesophagus, GOJ and cardia in one year. A standardised proforma was designed to capture demographics, clinico-pathological and endoscopic data including the relationship of tumour epicentre with the distal end of the tubular oesophagus, the presence or absence of Barrett's oesophagus; history of recurrent heartburn or regurgitation. To avoid reversed causality, we disregarded symptoms that occurred less than five years prior to cancer diagnosis.

Results 37 patients were diagnosed with adenocarcinoma of lower oesophagus and cardia between January and December 2011. 73.5\% of patients were male and the age at diagnosis ranged between 45 and 97 years. Only 32\% of diagnosed cancers were referred through 'Urgent suspected cancer' pathway. $43 \%$ of patients were smokers and $28 \%$ were ex-smokers; $55 \%$ drank alcohol regularly. Only 6 out of 37 patients had chronic symptoms (more than 5 years duration) suggestive of reflux including nausea, heartburn and sore tongue. $62 \%$ of these patients were on proton pump inhibitors or Histamine blockers at the time of diagnosis. $20 \%$ of the endoscopies showed a large hiatus hernia at index endoscopy and 20\% showed evidence of Barrett's (length between 6 and $11 \mathrm{~cm}$ ). Only $30 \%$ of patients were treated with curative intervention and the rest were managed by palliative means. $63.8 \%$ of diagnosed patients were not alive at one year of follow up out of which one patient had treatment with curative intent, Correlation testing between GORD and diagnosis of GOJ adenocarcinoma using regression analysis did not reach statistical significance.

Conclusion This interim report did not reveal a significant correlation between chronic reflux and development of gastrointestinal adenocarcinoma. The number is too small to permit a firm conclusion and we will report further results upon completion of the 10 years.

Disclosure of Interest None Declared.

\section{PWE-157 TWO DIMENSIONAL MAPPING OF MUTANT CLONES IN HUMAN COLONIC CRYPTS REVEAL STEM CELL DYNAMICS AND MIGRATION PATTERNS}

doi:10.1136/gutjnl-2013-304907.445

1."B Cereser, ${ }^{2} \mathrm{~A}-\mathrm{M}$ C Baker, ${ }^{2} \mathrm{P}$ J Tadrous, ${ }^{3} \mathrm{~A}$ Humphries, ${ }^{4} \mathrm{M}$ Novelli, ${ }^{4} \mathrm{M}$ Rodriguez-Justo, ${ }^{5} \mathrm{~T}$ A Graham, 'S A McDonald, 'N A Wright. 'Tumour Biology, Barts Cancer Institute, Barts and The London SMD, QMUL, London; ${ }^{2}$ Department of Histopathology, Northwick 